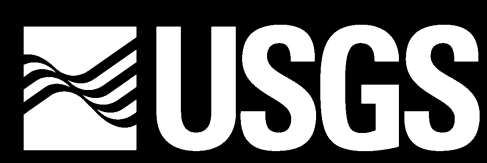

science for a changing world

Three-month

performance evaluation

of the Nanometrics, Inc.,

Libra Satellite

Seismograph System in

the Northern California

Seismic Network

by David H. Oppenheimer

Open-File Report 00-330

2000

This report is preliminary and has not been reviewed for conformity with U.S. Geological Survey editorial standards or with the North American Stratigraphic Code. Any use of trade, firm, or product names is for descriptive purposes only and does not imply endorsement by the U.S. Government.

U.S. DEPARTMENT OF THE INTERIOR

U.S. GEOLOGICAL SURVEY

${ }^{1}$ Menlo Park, California 


\section{Three-month performance evaluation of the Nanometrics, Inc., Libra Satellite Seismograph System in the Northern California Seismic Network}

\section{Background}

In 1999 the Northern California Seismic Network (NCSN) purchased a Libra satellite seismograph system from Nanometrics, Inc to assess whether this technology was a cost-effective and robust replacement for their analog microwave system. The system was purchased subject to it meeting the requirements, criteria and tests described in Appendix A. In early 2000, Nanometrics began delivery of various components of the system, such as the hub and remote satellite dish and mounting hardware, and the NCSN installed and assembled most equipment in advance of the arrival of Nanometrics engineers to facilitate the configuration of the system. The hub was installed in its permanent location, but for logistical reasons the "remote" satellite hardware was initially configured at the NCSN for testing.

During the first week of April Nanometrics engineers came to Menlo Park to configure the system and train NCSN staff. The two dishes were aligned with the satellite, and the system was fully operational in 2 days with little problem. Nanometrics engineers spent the remaining 3 days providing hands-on training to NCSN staff in hardware/software operation, configuration, and maintenance. During the second week of April 2000, NCSN staff moved the entire remote system of digitizers, dish assembly, and mounting hardware to Mammoth Lakes, California. The system was reinstalled at the Mammoth Lakes water treatment plant and communications successfully reestablished with the hub via the satellite on 14 April 2000. The system has been in continuous operation since then.

This report reviews the performance of the Libra system for the three-month period 20 April 2000 through 20 July 2000. The purpose of the report is to assess whether the system passed the acceptance tests described in Appendix A. We examine all data gaps reported by NCSN "gap list" software and discuss their cause.

\section{System configuration and effect on data loss}

We purchased the Libra system as a replacement for our analog microwave system that telemeters seismic data from Mammoth Lakes to Menlo Park. At the remote site in Mammoth Lakes we connected three Nanometrics 6-channel HRD24 dataloggers to a Cygnus satellite terminal and configured them in an unusual fashion. Rather than having one datalogger per station, we use each of the 6 channels of the datalogger to digitize a single, analog, vertical channel telemetered from a remote station. Thus with 3 data loggers at the remote site we were able to transmit with one transceiver and very small aperture terminal (VSAT) 100 sample/sec, 24-bit continuous data from 18 different seismic stations 
(Figure 1) over a single, $100 \mathrm{KHz}$ block of satellite transponder bandwidth. Nanometrics software permits each channel to be given a different name.

The Nanometrics acquisition software has the capability to retrieve missing data packets from the remote seismic datalogger as long as the data resides in the memory buffer of the datalogger. The length of time in which data resides in memory is a function of the data sample rate, number of channels, amount of datalogger memory, and efficiency of data compression. Practically speaking, if the satellite system is available and data compression is optimal, it can recover missing data within 1.5 hours of occurrence after which the data in memory is overwritten. Because the NCSN provides information about current earthquake locations and magnitudes to the public via the WWW, its Earthworm software is designed to operate under near real-time conditions. Therefore, we impose a maximum $30 \mathrm{sec}$ delay after receipt of a data packet before we broadcast the Nanometrics data to the NCSN Earthworm system using the NAQS2EW module (described at http://www.cnss.org/EWAB/toc.htm). This delay interval provides the Libra system sufficient time to make at least one request for missing packets, yet provides the Earthworm system with data in a timely fashion for rapid reporting of earthquakes. This report discusses data quality broadcast after the 30 -sec delay, which we hereafter refer to as " $30-\mathrm{sec}$ broadcast data". The ability of the Libra system to ultimately recover all missing data, albeit in a delayed fashion, facilitates NCSN needs for waveform archival. Except where noted, the Libra system ultimately recovered all missing data.

The analog signals have much lower dynamic range, $~ 40 \mathrm{~dB}$, than does the Nanometrics digitizer (nominally $132 \mathrm{~dB}$ ). Soon after installation, we realized that the analog input signal levels were not optimally matched to the digitizer, such that we were using most the dynamic range to digitize telemetry noise. The "noisy" signal reduced the efficiency of data compression, and most data samples compressed to 2 bytes rather than 1 under normal signal levels. Consequently, the digital signal utilized more of the satellite capacity than intended. This also reduced the amount of data available for retransmission in the datalogger buffer to 45 minutes. While poor data compression has the potential to degrade data throughput, we did not observe this during the threemonth interval because we budgeted satellite bandwidth for 2.4 bytes of data/sample based on a compression analysis of a M5 earthquake recorded at an epicentral distance of $7.5 \mathrm{~km}$ by the Berkeley Digital Seismic Network on a 24-bit datalogger with broadband sensors and accelerometers.

However, as discussed below, during a severe "rain" fade we lost communications with the remote system for 45 minutes. When communications was reestablished between the hub and the remote, there was insufficient satellite bandwidth for the Libra system to transmit both current data and "requested" data packets for the 45-minute interval before data was overwritten in datalogger memory. In future deployments we will attenuate the analog signals so that the level of the analog input signal is appropriately matched to the digitizer range. 


\section{Observations}

We illustrate "30-sec broadcast data " loss in two different ways. In Figure 2 we show the total data loss per day both in absolute seconds lost and as a percent of time lost per day. The latter metric is an acceptance specification shown in Appendix A and must be less than 1\%/day on average. In Figure 3 we show the same information, but as histograms that show the number of gaps/day of various gap length. A feature of Nanometrics equipment is that channel 1 receives priority transmission if throughput is inadequate. While this feature is observed in the performance of each datalogger, we show only the first channel of each datalogger (see Figure 1) because the plots for channels 2-6 are nearly identical.

In the following discussion we note when all significant system outages occurred. We note whether the outages were due to the performance of the Libra system or whether the NCSN took down the system.

\section{April 2000 - Rain Fade and Snow Storm}

This outage occurred the same day that the remote site was reinstalled in Mammoth Lakes. The outage is not shown in Figures 2 and 3 because outage log files only commence on 20 April 2000. Even though the outage precedes the 3-month evaluation period, we discuss the event because it illustrates a weakness common to all satellite communication systems. Communication between the hub and remote was lost between the hours of 18:30 and 21:00. The onset of the fade was sudden and caused by a large thundercloud that passed overhead in Menlo Park. The duration lasted 45 minutes. NCSN staff still in Mammoth Lakes, where it was sunny, confirmed that data acquisition there was unimpeded. Due to confusion about why the system lost connectivity, the NCSN rebooted the Carina, causing yet an additional 11 minutes of outage.

As the cloud sailed out of the path to the satellite, data resumed and throughput was $100 \%$. The system then began requesting retransmission of data in the rain fade interval. Only channel 1 of each datalogger had nearly complete data for the fade interval. About $45 \%$ of that data was retransmitted. All other channels had 500-700 sec (out of 3600) still missing, in lots of small gaps. What data that was received was $\sim 30-35 \%$ retransmitted. Subsequent analysis of the system performance revealed that data recovery through retransmission requests was impeded by the poor compression performance discussed above. We expect that data recovery will greatly improve when we attenuate the input analog signal level so that the analog data compresses to 1 byte.

No other weather-related outages were observed in the 3-month interval. In particular, there was a brief snowstorm at Mammoth Lakes earlier on 14 April 2000, and no data loss was observed.

\section{April 2000 - Move of Carina transceiver}

At 20:00 while the Libra system was operational, we dragged the cabinet hardware containing the Carina transceiver a few feet across the floor, closed its back door, then again pushed it back to the same position. The Carina hub 
stopped receiving any data at 20:09 by the system clock and automatically rebooted at $21: 15$ as programmed. It again failed to reestablish connectivity with the Cygnus. Signal level at the dish was verified to be ok. At 22:12 the NCSN staff performed a power-down on the Carina, disconnected and reconnected all cables, and restored power to the unit at 22:27. System still failed to establish connectivity with remote, so NCSN staff performed a software shutdown/reboot, and again verified that signal level at dish was ok. At 22:54 the Carina reestablished connectivity with remote. The system was off the air for approximately 2:46 (see Figures 2 and 3).

While we are unable to verify if the movement of the transceiver cabinet caused the system outage, the near coincidence and uniqueness of the event indicates a cause and effect. Nanometrics staff attempted to replicate the situation and was unsuccessful. They speculate that a cable may have a marginal connection. The NCSN has not tried to replicate the situation during the test period because we plan to move the Carina in September and will attempt to replicate the failure mode at that time.

26 June 2000

The NCSN shut down the Carina for approximately 20 minutes to move the cabinet.

\section{July 2000}

The power was off at the Mammoth Lakes Water Treatment facility for 73 minutes. All 3 data loggers and satellite system went down and automatically rebooted when power was restored. This was an unrecoverable data loss due to lack of a UPS.

\section{Malfunctioning HRD24}

Datalogger HRD1 (Figure 1) immediately malfunctioned after installation at Mammoth Lakes. The unit periodically rebooted, causing permanent data loss as shown in Figure 2 and the large numbers of gaps in Figure 3. The unit completely died on 13 May 2000 and was replaced on 24 May 2000. During this 11-day interval the "total amount of data lost per day" (Figure 2) is meaningless for HRD1. After the unit was replaced no further gaps occurred. These data gaps are not related to Libra performance.

\section{Unexplained gaps}

On 16 June 2000 a series of gaps as small $1 \mathrm{sec}$ and exceeding $4 \mathrm{sec}$ occurred, resulting in some disruption to near real-time (30 sec delay) broadcast of data (Figures 2 and 3). Approximately $1350 \mathrm{sec}$ of data were delayed, but the data were ultimately recovered by retransmission requests. There was no indication in NCSN notes of any system maintenance activity on that day. Similar gaps occurred on 23 June 2000. These delayed transmissions may be weather related. 


\section{Discussion}

The procurement by the NCSN of a satellite based seismic telemetry system specified requirements and evaluation criteria (Appendix A) that any vendor, including Nanometrics, must meet. The Nanometrics system, as purchased, met all of these requirements. The procurement also included three acceptance tests to be evaluated after 3 months of operation. In this section we discuss the performance of the Nanometrics Libra system with regard to these tests.

1) The data packet loss for initial transmission (after $30 \mathrm{sec}$ ) exceeded $1 \%$ per day on three days - 14 April due to rain fade, 20 April when the Carina was offline, and on 16 June 2000. On average, however, the initial data packet loss was near zero and the system meets the test.

2) The final data packet loss within the time interval available for retransmission (i.e., $\sim 1.5$ hour) did not exceed $0.01 \%$ per day except for the rain fade of 14 April and when the Carina was offline on 20 April 2000. In retrospect, this acceptance test is vague. The final packet loss is also a function of the amount of satellite bandwidth available for real-time and retransmit requests. If the amount satellite bandwidth leased were sufficient only to carry real-time data, then no system would be able to deliver retransmit requests.

Conversely, if an "infinite" amount of bandwidth were available, then the test would be meaningless.

When we specified the satellite bandwidth, we did not anticipate that the digitization of analog system noise would compromise data compression. We now believe that if we appropriately attenuate the analog signal level to match the dynamic range of the digitizer that the satellite bandwidth we currently lease will be sufficient to will meet this test. Otherwise, we will allocate more satellite bandwidth. In any event, the Libra system performed as designed, given the available bandwidth on the satellite channel.

Regarding rain fade, in a VSAT system the manufacturer is responsible for insuring that its equipment meets specifications and is reliable. The space segment provider is responsible for preparing a link budget that guarantees a certain level of availability, in this case $99.9 \%$. The most common solution to this problem is to negotiate more power on the satellite and thus gain more rain fade margin. We can also install more memory at the remote site to improve ultimate data recovery. A third and more costly approach to overcome the problem of downlink fade is to have a second hub at a second location.

3) No large earthquakes occurred during the 3-month evaluation period. Consequently, no evaluation is possible.

\section{Conclusion}

During the evaluation period we observed two occasions where telemetry outages can be attributed to the Libra system. The first outage occurred on the day when the system was installed in Mammoth Lakes. That outage was due to 
"rain" fade, a weather phenomenon that affects all satellite telemetry systems. We believe we can reduce the likelihood of rain fades by increasing the transmit power to the satellite. The second outage occurred when the hub transceiver was moved slightly while the system was in operation. The latter outage has not been explained and may be due to a loose connection in the cabling. Otherwise, the system performed as advertised and data recovery under typical operating conditions met specifications. Other outages did occur, but we can attribute them to causes other than the Libra system.

Based on this three-month evaluation period, the Libra system appears to be a very reliable means of digital telemetry, can be deployed in remote locations in a few days, and offers a cost-effective solution to replacing the obsolete NCSN microwave system.

\section{Acknowledgements}

Many people deserve thanks for making this project a success. Lynn Dietz deserves a great amount of credit for writing and supporting the data interface from the Libra system to our Earthworm system, configuring system hardware, troubleshooting and logging system performance, and managing the system on a daily basis. Likewise, Tom Burdette managed the complex installation of the physical hardware, such as king posts, dishes, and cabling in both Menlo Park and Mammoth Lakes. Gray Jensen participated in most aspects of the project, including system procurement, design of the analog-to-digital configuration at the remote end, and physical hardware installation. Bob McClearn, Lester Sutherland, Fred Fischer, Dave Reneau, Dave Croker, and Don Farrell installed equipment, aligned dishes, and troubleshot equipment. Will Kohler assembled and configured computers and networking for the installation. Don Ritchey assembled the VCO hardware for the analog-to-digital rack at Mammoth Lakes. Brad Tavner and Emil Farkas of Nanometrics provided on-site installation instructions, training, and follow-on support. Thanks to Lynn Dietz, Will Kohler, Tom Burdette, and Gray Jensen for their reviews of the report. 
Appendix A. Procurement requirements and evaluation criteria

\section{Requirements}

The Northern California Seismic Network (NCSN) seeks to procure a system utilizing digital satellite telemetry to replace its analog microwave telemetry system. The system must be similar to or exceed the capabilities of the Libra Satellite Seismograph system developed by Nanometrics, Inc. The system must have the following features:

- All data must be continuously transmitted directly between the field VSAT, satellite, and the central data acquisition receive site (to be deployed in Menlo Park, CA) and not utilize any terrestrial communications lines (e.g., Internet or private telephone circuits) or multiple satellite hops.

- The NCSN has a substantial investment in Nanometrics HRD24 seismic dataloggers and wishes to make continued use of this investment. Software at VSAT must interface directly to a Nanometrics HRD24 datalogger and be able to request that the dataloggers re-transmit lost or corrupt data packets for the amount of data residing in the unit's memory.

- The system must be able to integrate at a single VSAT digital data from multiple, physically discrete seismic dataloggers, including those local to the VSAT, and that may be digitizing data at different sample rates and that may have different numbers of channels at each datalogger.

- Multiple VSAT units must be capable of sharing a single satellite link for transmitting their data to the central receive site.

- The central-site data acquisition software must function under a Solaris or NT operating system. Software must be able to broadcast continuously acquired seismic data within $1 \mathrm{sec}$ in the USGS Earthworm UDP packet format, regardless of success of data transmission.

- The system must transmit data with maximum delay (from time of sample acquisition to receipt of sample by central-site acquisition software) no greater than $6 \mathrm{sec}$.

- The central-site data acquisition software must be able to acquire delayed data packets due to retransmit requests of lost/corrupted information, and provide that data, on request, in the proper time order to the USGS Earthworm system.

- The integrated software and hardware system must be in commercial production and in use by customers. The NCSN will not accept any hardware or software products in development.

- The system must be gradually expandable, such that ultimately the centralsite acquisition could receive and re-broadcast data to the USGS Earthworm system at rates of approximately $2 \mathrm{Mbytes} / \mathrm{sec}$. 
- The system must be able to operate $-20^{\circ} \mathrm{C}$ to $+55^{\circ} \mathrm{C}$ with less than $30 \mathrm{~W}$ power consumption, including VSAT, remote digitizer, and GPS

\section{Evaluation criteria}

- Efficiency. A premium will be placed on systems that maximize bandwidth efficiency and minimize the number of VSAT's/hubs/space segments to transmit NCSN seismic data.

- Timely data delivery. While a premium is placed on efficient utilization of satellite space segment bandwidth, systems utilizing seismic compression algorithms to achieve higher telemetry bandwidth must be able to specify the anticipated delay in data transmission if compression is minimal during large earthquakes. The NCSN will provide a benchmark suite of seismograms to prospective vendors for reporting on the resulting amount (or lack thereof) of compression. The NCSN will take into consideration the degradation of compression during large earthquakes in evaluating the efficiency of data delivery.

- Ease of operation. A premium will be placed on telemetry systems that allow remote configuration of the VSAT's via the satellite. We will also place a premium on systems that allow remote monitoring of VSAT equipment status, such as temperature, supply voltage, and data integrity.

- Cost

- Timely delivery of hardware and software

\section{Acceptance tests}

The following tests will be carried out. The criteria will be evaluated at the end of a 3-month operation period.

1. Subject to satellite availability (i.e., excluding rain fade, solar storm outages, satellite failure, etc.), data packet loss in initial transmission may not exceed, on average $1 \%$ per day for the entire space segment. Software gaps will be identified with NCSN "gap list" software or by software mutually agreed upon by the NCSN and vendor.

2. Final data packet loss within time interval available for retransmission from a 4 Mbyte memory buffer shall not exceed $0.01 \%$ per day for the entire space segment.

3. The system transmission throughput cannot significantly degrade during large or extended earthquake sequences. Should data transmission delays exceed time interval in digitizer buffer resulting in a permanent loss of data, the system will be considered to have failed to meet acceptance tests. Vendor is expected to supply NCSN with worst-case compression efficiency from NCSN-supplied benchmark seismograms in order to configure system with appropriate bandwidth per VSAT. 


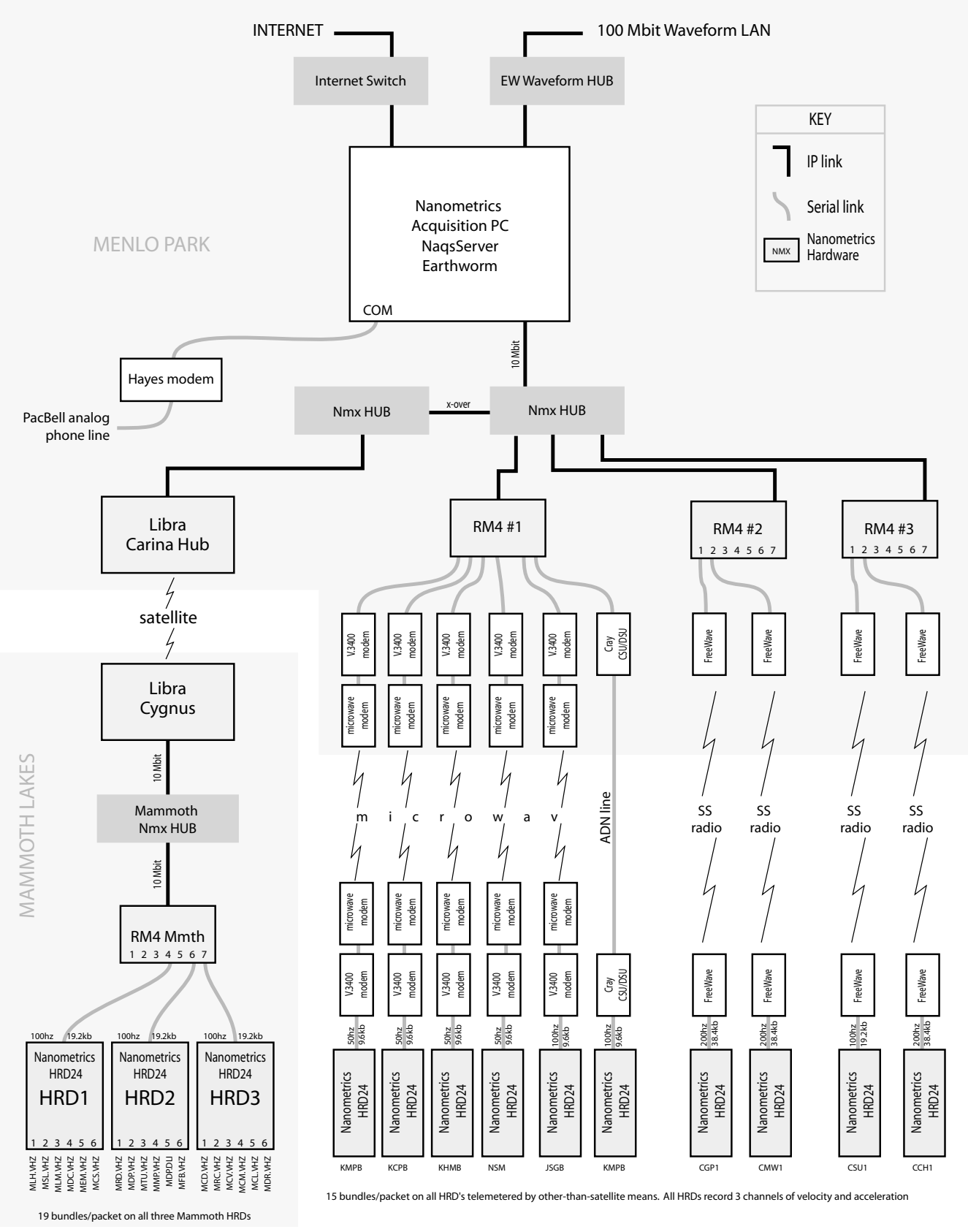

Figure 1. Nanometrics configuration in the NCSN. Shaded block at lower left hand side represents configuration of Cygnus hardware, networking, and data loggers at Mammoth Lakes. Shaded block in upper half of diagram represents Carina hardware, networking, and acquisition computers at the NCSN. 

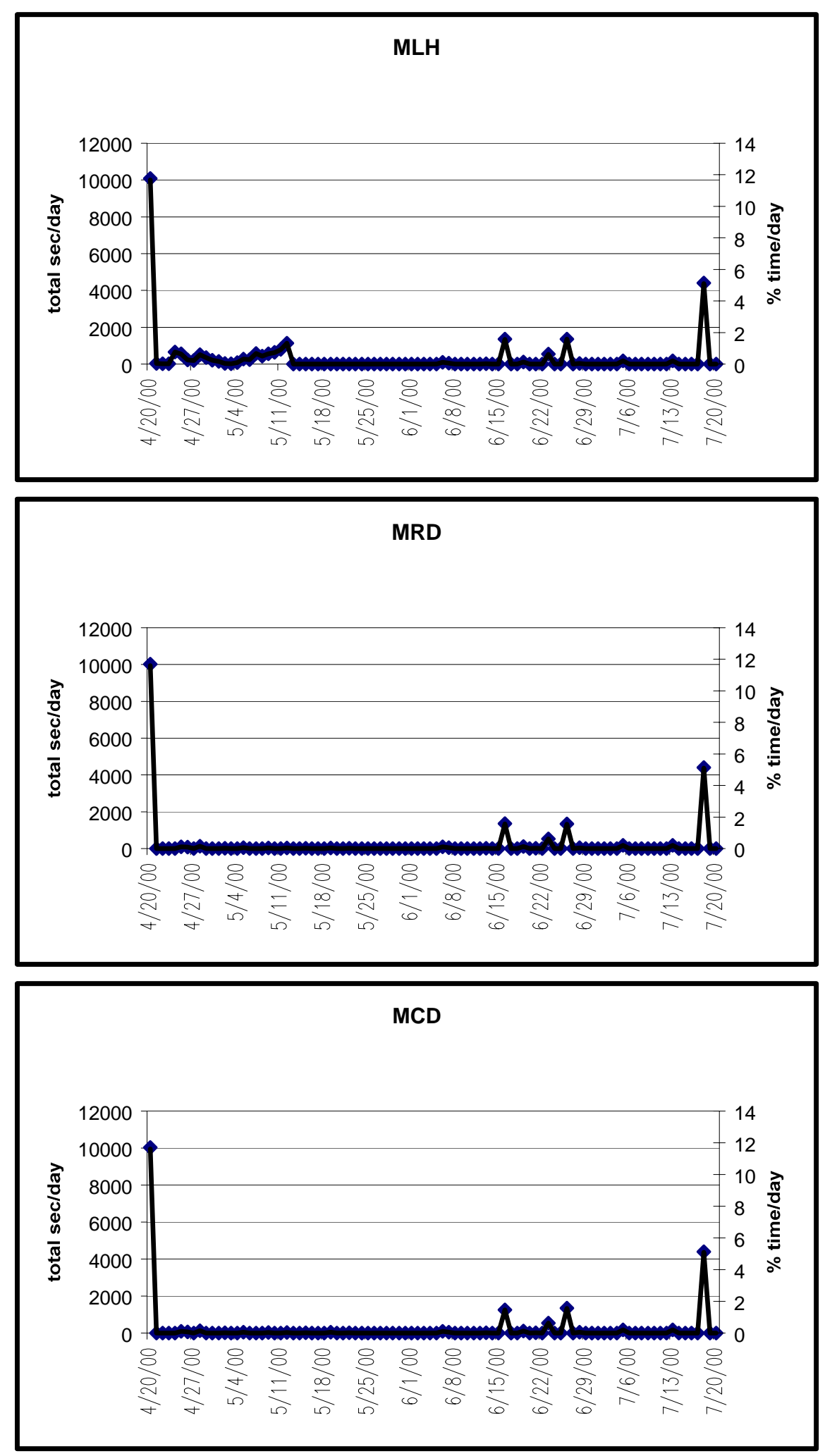

Figure 2. Total amount of data lost per day for "30-sec" broadcast data. Only channel 1 of each datalogger is shown. 

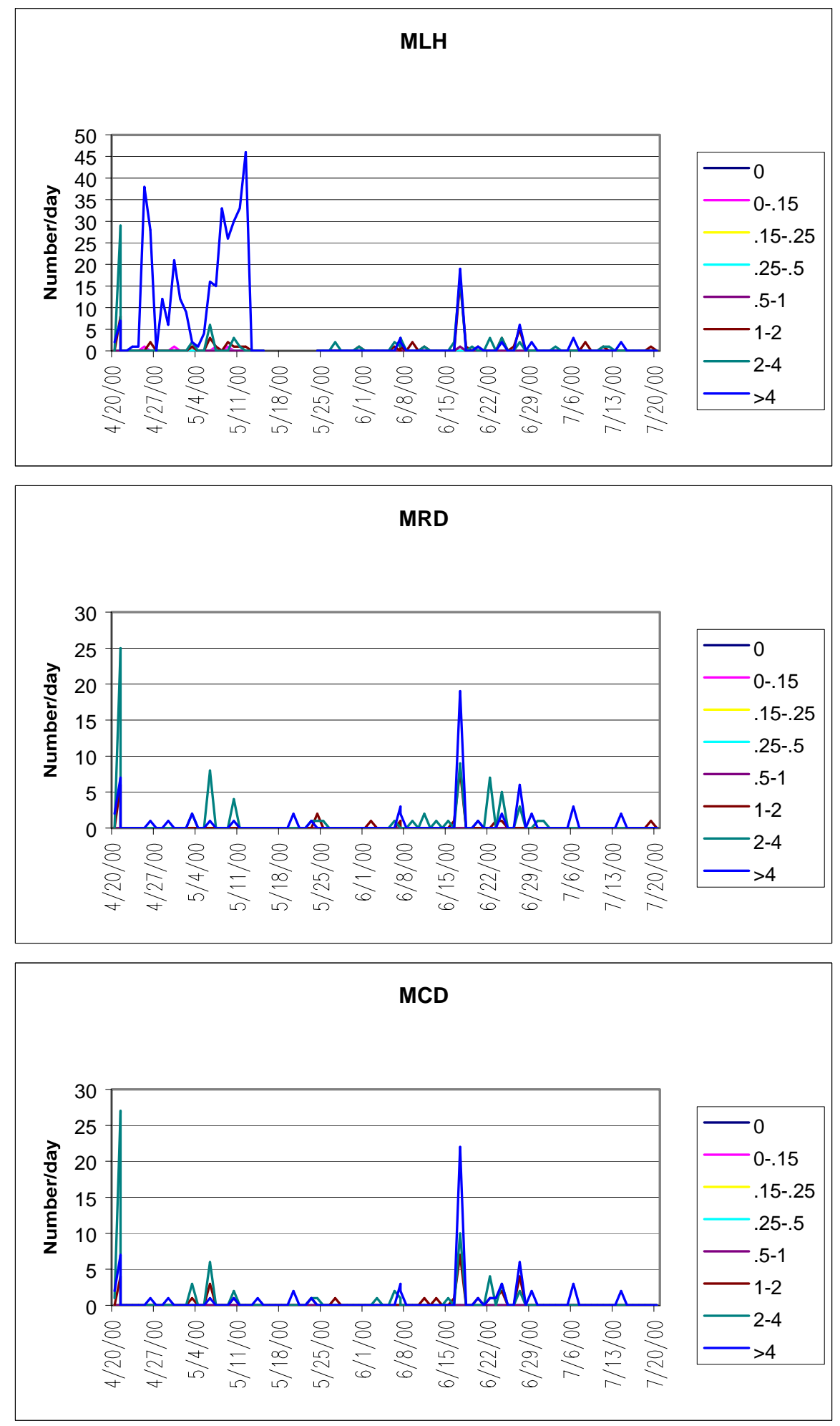

Figure 3. Histograms of data gaps as a function of gap length for "30-sec" broadcast data. Only channel 1 of each datalogger is shown. 